\title{
Indoleamine 2,3-Dioxygenase Activity Is Increased in Myelodysplastic Syndrome Patients
}

\author{
Kimihiro Yamaguchi ${ }^{1}$, Soranobu Ninomiya ${ }^{1, *} \mathbb{C}$, Takuro Matsumoto ${ }^{1}$, Nobuhiko Nakamura ${ }^{1}$ (D), \\ Hiroshi Nakamura ${ }^{1}$, Junichi Kitagawa ${ }^{1}$, Nobuhiro Kanemura ${ }^{1}$, Takeshi Hara ${ }^{1,2}$, \\ Suwako Fujigaki ${ }^{3}$, Yasuko Yamamoto ${ }^{3}$, Kuniaki Saito ${ }^{3}$, Hisashi Tsurumi ${ }^{1,2}$ and \\ Masahito Shimizu ${ }^{1}$ (D) \\ 1 First Department of Internal Medicine, Gifu University Graduate School of Medicine, Gifu 501-1194, Japan; \\ chris_kimihiro@yahoo.co.jp (K.Y.); matsutakeseven@yahoo.co.jp (T.M.); nnakamura-gif@umin.ac.jp (N.N.); \\ ayapokopoko2@gmail.com (H.N.); jkitagawa1128@gmail.com (J.K.); nkane@orion.ocn.ne.jp (N.K.); \\ haratake@muh.biglobe.ne.jp (T.H.); htsuru@gifu-u.ac.jp (H.T.); shimim-gif@umin.ac.jp (M.S.) \\ 2 Department of Hematology, Matsunami General Hospital, Gifu 501-6062, Japan \\ 3 Department of Disease Control and Prevention, Fujita Health University Graduate School of Health Science, \\ Toyoake 470-1192, Japan; soranobu2000@gmail.com (S.F.); yama-y@fujita-hu.ac.jp (Y.Y.); \\ saitok@fujita-hu.ac.jp (K.S.) \\ * Correspondence: sxninomi@gifu-u.ac.jp; Tel.: +81-58-230-6308; Fax: +81-58-230-6310
}

Received: 20 October 2020; Accepted: 10 December 2020; Published: 11 December 2020

\begin{abstract}
Tryptophan (TRP) metabolism via the indoleamine 2,3-dioxygenase (IDO) subset of the kynurenine (KYN) pathway is one of the most important mechanisms of immune escape in cancer. TRP is converted into several biologically active KYN metabolites. However, the role of KYN metabolic products and related enzymes has not been clarified in patients with hematological malignant tumors. Here, we examined the serum concentrations of TRP, KYN, and the KYN metabolites kynurenic acid, anthranilic acid, and 3-hydroxyanthranilic acid in 157 patients stratified into five different hematological malignant tumors. KYN was the most abundant product of the TRP metabolic pathway among all five diagnostic categories. Serum KYN was increased in myelodysplastic syndrome (MDS) patients. The KYN/TRP ratio was significantly higher in MDS patients than in acute myeloid leukemia patients. In conclusion, IDO activity is increased in MDS patients, and IDO inhibitors might represent a new therapeutic approach for MDS treatment.
\end{abstract}

Keywords: tryptophan metabolism; IDO; MDS

\section{Introduction}

Tryptophan (TRP) metabolism via indoleamine 2,3-dioxygenase (IDO) in the kynurenine (KYN) pathway is one of the most important mechanisms of tumor immune escape [1]. IDO is involved in the first step of TRP metabolism, which produces KYN and regulates biological processes that include host-microbiome signaling, immune cell response, and neuronal excitability [2]. These TRP metabolites inhibit antigen-specific T-cell proliferation and induce T-cell death [3]. Pro-inflammatory cytokines, such as interferon (IFN)- $\gamma$, increase the expression of IDO in many human cell types and tumor cells [3]. In turn, TRP metabolism mediated by immunoregulatory IDO helps tumors to escape from host immune responses and confers survival advantages on tumors [4]. High expression levels of IDO and increased serum KYN have been shown to worsen many types of cancer, including lymphoma and leukemia [5]. We have previously demonstrated the importance of assessing the levels of serum KYN or tumor tissue IDO as prognostic factors in diffuse large B-cell lymphoma (DLBCL) patients [5,6].

KYN is a major component of the TRP metabolic pathway and a key signaling molecule with immunosuppressive properties. KYN is converted into several biologically active secondary 
metabolites [7]. KYN is a common substrate for the formation of kynurenic acid (KYNA), anthranilic acid (AA), and 3-hydroxyanthranilic acid (3-HAA), which are catalyzed by kynurenine aminotransferase, kynureninase, and kynurenine-3-monooxygenase, respectively. AA is metabolized to 3-HAA, which is further metabolized to nicotinamide or picolinic acid via several enzymatic reactions. Metabolic changes in the KYN pathway have been investigated in the field of psychiatry [8]. Darlington et al. reported that AA plasma concentrations are higher in depressive patients than in healthy controls [7]. High levels of AA and low levels of TRP were also observed in the serum of depressive patients [9]. Despite recent extensive efforts, the role of KYN metabolic products and their enzymatic activities has not been clarified in patients with hematological malignant tumors.

This study aimed to examine the serum TRP-KYN metabolite profile in hematological malignant tumors.

\section{Materials and Methods}

\subsection{Patients and Blood Sample Collection}

The design of the retrospective case series was approved by the institutional review board at Gifu University Hospital (authorization number 2018-025). This study was performed in accordance with the Declaration of Helsinki. Every effort was made to protect patient confidentiality and personal information. We investigated 157 patients with myeloid leukemia (AML, $\mathrm{n}=25)$, myelodysplastic syndrome (MDS, $n=19)$, multiple myeloma (MM, $n=24)$, DLBCL $(n=65)$, and follicular lymphoma $(\mathrm{FL}, \mathrm{n}=24)$ who were admitted to our hospital from January 2014 to December 2016. Patients were diagnosed according to the World Health Organization (WHO) classification of hematopoietic tumors. Patient serum samples were taken on admission before any treatment and stored at $-20^{\circ} \mathrm{C}$. Patients were not on any special diet.

\subsection{Serum TRP Metabolite Measurements}

Serum concentrations of TRP, KYN, KYNA, AA, and 3-HAA were measured using high-performance liquid chromatography (HPLC). Each concentration is expressed as the molar concentration (mol/L (M)). Serum samples were mixed with $10 \%$ perchloric acid (4:1) and centrifuged at $14,000 \mathrm{rpm}$ for $10 \mathrm{~min}$ at $4{ }^{\circ} \mathrm{C}$. Next, the supernatant was used for quantification using the HPLC instrument. The mobile phase consisted of $10 \mathrm{mM}$ sodium acid containing $1.7-2.0 \%$ acetonitrile. The flow rate was $0.8 \mathrm{~mL} / \mathrm{min}$. The analytical column was a TSK gel ODS-100v (TOSOH, Tokyo, Japan; $4.6 \mathrm{~mm} \times 150 \mathrm{~mm}$ with a particle size of $3 \mu \mathrm{m}$ ). TRP and KYN were measured using a photodiode array detector (SPD-M30A; Shimadzu, Kyoto, Japan) at 280 and $365 \mathrm{~nm}$, respectively. AA, 3-HAA, and KYNA were measured using a fluorescence detector (RF-20Axs; Shimadzu, Kyoto, Japan) at an excitation wavelength of $320 \mathrm{~nm}$ and an emission wavelength of $420 \mathrm{~nm}$ for AA and 3-HAA and an excitation wavelength of $334 \mathrm{~nm}$ and an emission wavelength of $380 \mathrm{~nm}$ for KYNA. A calibration curve for these metabolites was performed. The value of the correlation coefficient (R2) was $>0.99$ within the tested concentration range (TRP: 97.9-3.1 $\mu \mathrm{M}$; KYN: 3.84-0.1 $\mu \mathrm{M}$; 3-HAA: 163.3-5.1 nM; KYNA: 132.1-4.1 nM; AA: 72.9-2.3 nM). The limit of detection is the lowest metabolite concentration in a sample that is positively identified against the background. The limit of quantification is the lowest metabolite concentration that is accurately measured under the analysis conditions. The precision of the method was tested by performing 10 independent intraday replicate measurements of the standard at 3 different concentrations. All values were within the acceptable concentration range of the standard percent coefficient of variation $(\% \mathrm{CV})<5$. Accuracy was determined using the recovery test, which confirmed that HPLC could be used to analyze biological samples (95-102\%).

\subsection{Statistical Analyses}

All statistical analyses were performed using EZR (Saitama Medical Center, Jichi Medical University, Saitama, Japan), a graphical user interface for $\mathrm{R}$ (The $\mathrm{R}$ foundation for statistical computing, 
Vienna, Austria). Normal distribution of data was assessed using the Kolmogorov-Smirnov test. If a normal distribution was followed, the mean and standard deviation were listed. If not, the median and interquartile range were listed. The concentration of serum TRP metabolites is presented as median and range. Nominal variables and categorical variables indicate the number of cases and percentages. Student's $t$-test was used for comparisons between two unpaired groups for normally distributed data. Welch's t-test was used for unequally distributed data. The Kruskal-Wallis test was used for comparisons among 5 hematological malignancies. Fisher's exact test was used to compare categorical variables. A value of $p<0.05$ was considered statistically significant.

\section{Results}

\subsection{Concentrations of Serum TRP Metabolites in Hematological Malignant Tumors}

We determined the serum levels of TRP, KYN, KYNA, AA, and 3-HAA in 157 patients with hematological malignant tumors (Table 1). Of the 157 patients enrolled in this study, 94 were male and 63 were female (range of age, 15-93 years; median age, 67 years). Patients were classified as having AML $(n=25)$, MDS $(n=19), \operatorname{MM}(n=24)$, DLBCL $(n=65)$, and FL $(n=24)$. The median serum concentration of KYN in 157 samples from hematological patients was $1.99(0.44-23.57) \mu \mathrm{M}$, which was significantly higher than that of AA (69.69 (0.37-943.19) nM), KYNA (34.04 (3.65-1498.81) $\mathrm{nM}$ ), and 3-HAA (3.21 (0.03-50.48) nM). Serum levels of 3-HAA were significantly lower than serum levels of AA and KYNA. There was no significant difference between serum levels of AA and KYNA. We calculated the ratio of the metabolites to estimate the activity of the enzymes in the metabolic pathway. The details regarding age and gender are summarized in Table 2. The concentration of serum TRP was significantly lower in females $(41.96(8.94-91.06) \mu \mathrm{M})$ than in males (47.19 (15.63-88.62) $\mu \mathrm{M})$. The concentration of serum KYN was significantly lower in females $(1.81(0.44-10.34) \mu \mathrm{M})$ than in males $(2.12(0.71-23.57) \mu \mathrm{M})$. However, the ratio of serum KYN/TRP was not different between male and female patients. The serum concentrations of KYN, KYNA, and 3-HAA were significantly higher in older patients than in younger patients. There were no significant differences in serum TRP levels between younger patients and older patients. The ratios of serum KYN/TRP were higher in older patients than in younger patients.

\subsection{Differences in the Concentrations of TRP Metabolites According to Diagnostic Categories}

Table 3 shows serum levels of TRP metabolites categorized into five hematological malignant tumors. Serum levels of KYN were significantly higher than those of AA, KYNA, and 3-HAA among all malignant tumors. Serum levels of KYN in MDS patients were significantly higher when compared to those in AML patients (Figure 1). The KYN/TRP ratio was significantly higher in MDS patients than in AML patients. There were no significant differences in serum AA, KYNA, and 3-HAA levels between MDS and AML patients. The serum levels of KYN in MDS patients were higher than in patients with any of the other conditions. There were no significant differences in age, gender, liver function, and renal function between MDS and AML patients (Table 4). 
Table 1. Concentrations of tryptophan metabolites and relative ratios of the metabolites in 157 patients with hematological malignancies.

\begin{tabular}{lc}
\hline \multicolumn{1}{c}{ Total } & $\mathbf{n}=\mathbf{1 5 7}$ \\
\hline Age & $67(15-93)$ \\
males & $94(60 \%)$ \\
Tryptophan $(\mu \mathrm{M})$ & $45.84(8.943-91.06)$ \\
Kynurenine $(\mu \mathrm{M})$ & $2.00(0.44-23.57)$ \\
Kynurenic acid $(\mathrm{nM})$ & $32.98(3.65-1498.91)$ \\
Anthranilic acid $(\mathrm{nM})$ & $74.89(0.37-943.19)$ \\
3-Hydroxyanthranilic acid $(\mathrm{nM})$ & $3.19(0.33-50.48)$ \\
Kynurenine/Tryptophan ratio & $0.047(0.017-0.473)$ \\
Kynurenic acid/Kynurenine ratio & $16.34(1.59-374.52)$ \\
3-Hydroxyanthranilic acid/Kynurenine ratio & $1.64(0.10-14.45)$ \\
Anthranilic acid/Kynurenine ratio & $35.2(0.19-687.05)$ \\
3-Hydroxyanthranilic acid/Anthranilic acid ratio & $0.041(0.001-2.277)$ \\
\hline
\end{tabular}

Table 2. Comparison of tryptophan metabolites by gender and age.

\begin{tabular}{|c|c|c|c|c|c|c|}
\hline & \multicolumn{2}{|c|}{ Gender } & \multirow[b]{2}{*}{$p$} & \multicolumn{2}{|c|}{ Age } & \multirow{2}{*}{$p$} \\
\hline & males $(n=94)$ & females $(n=63)$ & & $<60(\mathrm{n}=42)$ & $\geq 60(n=115)$ & \\
\hline $\mathrm{TRP}(\mu \mathrm{M})$ & $47.46(15.63-88.62)$ & $41.43(8.94-91.06)$ & $<0.001$ & 47.49 (20.61-91.06) & $45.83(8.94-88.62)$ & 0.679 \\
\hline $\mathrm{KYN}(\mu \mathrm{M})$ & $2.10(0.71-23.57)$ & $1.82(0.44-10.34)$ & 0.083 & $1.71(0.44-4.84)$ & $2.11(0.60-23.57)$ & 0.025 \\
\hline KYNA (nM) & $34.37(3.65-1498.91)$ & $32.25(13.36-278.09)$ & 0.368 & $29.58(11.05-272.58)$ & $35.84(3.65-1498.91)$ & 0.034 \\
\hline $\mathrm{AA}(\mathrm{nM})$ & $78.04(5.90-943.19)$ & $70.12(0.37-583.99)$ & 0.933 & $64.15(0.37-391.07)$ & $76.92(12.16-943.19)$ & 0.237 \\
\hline 3-HAA (nM) & $3.29(0.27-50.48)$ & $2.78(0.33-20.95)$ & 0.226 & $2.58(0.33-13.43)$ & $3.49(0.27-50.48)$ & 0.003 \\
\hline KYN/TRP & $0.04(0.02-0.47)$ & $0.05(0.02-0.35)$ & 0.727 & $0.04(0.02-0.16)$ & $0.05(0.02-0.47)$ & 0.011 \\
\hline KYNA/KYN & $15.77(1.59-374.52)$ & $16.61(6.59-110.33)$ & 0.404 & $16.12(5.42-304.55)$ & $16.48(1.59-374.52)$ & 0.736 \\
\hline 3-HAA/KYN & $1.72(0.19-14.45)$ & $1.53(0.10-8.19)$ & 0.658 & $1.50(0.10-9.44)$ & $1.72(0.10-14.45)$ & 0.078 \\
\hline $\mathrm{AA} / \mathrm{KYN}$ & $32.13(4.14-628.80)$ & $41.84(0.19-687.05)$ & 0.220 & $43.81(0.19-269.59)$ & $33.02(4.60-687.05)$ & 0.687 \\
\hline 3-HAA/AA & $0.049(0.001-2.277)$ & $0.034(0.002-1.000)$ & 0.300 & $0.028(0.002-2.277)$ & $0.049(0.001-1.059)$ & 0.187 \\
\hline
\end{tabular}

TRP: tryptophan; KYN: kynurenine; KYNA: kynurenic acid; AA: anthranilic acid; 3-HAA: 3-hydroxyanthranilic acid. 
Table 3. The concentrations of serum tryptophan metabolites and their ratios in the 5 diagnostic categories of hematological malignancies.

\begin{tabular}{|c|c|c|c|c|c|c|}
\hline Diagnosis & AML (n = 25) & MDS (n = 19) & $\mathrm{MM}(\mathrm{n}=24)$ & DLBCL $(n=65)$ & FL $(n=24)$ & $p$ \\
\hline Age & $63(17-83)$ & $72(15-89)$ & $67(45-87)$ & $70(2793)$ & $63(49-83)$ & 0.064 * \\
\hline Males & $16(64 \%)$ & $14(74 \%)$ & $11(46 \%)$ & $41(63 \%)$ & $11(46 \%)$ & $0.30 * *$ \\
\hline Tryptophan $(\mu \mathrm{M})$ & 50.9 (20.61-79.07) & $50.34(17.51-67.81)$ & $37.95(8.94-63.26)$ & $42.96(15.63-88.62)$ & $46.06(22.02-91.06)$ & $0.012 *$ \\
\hline Kynurenine $(\mu \mathrm{M})$ & $1.68(0.84-4.09)$ & $3.03(1.36-23.57)$ & $1.44(0.85-11.53)$ & $2.12(0.60-8.24)$ & $2.26(0.44-2.91)$ & $<0.01^{*}$ \\
\hline Kynurenic acid (nM) & $35.3(14.38-86.52)$ & $39.06(15.21-1498.91)$ & $26.22(4.00-278.09)$ & $32.87(12.72-344.56)$ & $32.29(3.65-53.83)$ & 0.17 * \\
\hline Anthranilic acid (nM) & $46.78(14.85-943.19)$ & $63.23(0.37-385.80)$ & $59.97(18.99-583.99)$ & $95.72(5.90-639.49)$ & $47.53(11.90-391.07)$ & $0.52 *$ \\
\hline 3-Hydroxyanthranilic acid (nM) & $2.38(0.41-13.07)$ & $3.04(0.37-50.48)$ & $2.18(0.27-10.59)$ & $3.44(0.33-23.34)$ & $4.36(0.61-24.99)$ & $0.021 *$ \\
\hline KYN/TRP & $0.03(0.02-0.09)$ & $0.05(0.03-0.37)$ & $0.04(0.02-0.35)$ & $0.05(0.02-0.47)$ & $0.04(0.02-0.08)$ & 0.052 * \\
\hline KYNA/KYN & $18.62(5.97-37.49)$ & $15.57(5.42-63.58)$ & $16.40(3.63-304.55)$ & $16.24(5.00-374.52)$ & 16.35 (1.59-110.33) & 0.43 * \\
\hline 3-HAA/KYN & $1.17(0.26-7.67)$ & $1.68(0.19-4.39)$ & $1.32(0.25-5.15)$ & $1.72(0.10-14.45)$ & $1.92(0.26-11.04)$ & 0.39 * \\
\hline $\mathrm{AA} / \mathrm{KYN}$ & $27.91(9.20-628.80)$ & $20.66(0.19-94.30)$ & 35.09 (7.17-687.05) & $46.51(4.14-255.54)$ & $29.33(5.01-1500.85)$ & 0.013 * \\
\hline 3-HAA/AA & $0.042(0.001-0.577)$ & $0.057(0.007-1.000)$ & $0.037(0.004-0.495)$ & $0.036(0.002-2.277)$ & $0.082(0.007-1.059)$ & 0.54 * \\
\hline
\end{tabular}

AML: acute myeloid leukemia; DLBCL: diffuse large B-cell lymphoma; FL: follicular lymphoma; MDS: myelodysplastic syndromes; MM: multiple myeloma. TRP: tryptophan; KYN: kynurenine; KYNA: kynurenic acid; AA: anthranilic acid; 3-HAA: 3-hydroxyanthranilic acid. *: Kruskal-Wallis test. ${ }^{* *}$ : Fisher's Exact Test.

Table 4. Comparison of clinical characteristics of patients with acute myeloid leukemia and myelodysplastic syndrome patients.

\begin{tabular}{cccc}
\hline Diagnosis & AML $(\mathbf{n}=\mathbf{2 5})$ & MDS $(\mathbf{n}=\mathbf{1 9})$ & $p$ \\
\hline Age & $59(17-83)$ & $67(15-89)$ & 0.144 \\
Males & $16(64 \%)$ & $14(74 \%)$ & 0.53 \\
Total bilirubin (mg/dL) & $0.7(0.1-1.3)$ & $0.9(0.3-2.8)$ & 0.33 \\
Aspartate transaminase (IU/L) & $23(11-55)$ & $23(6-49)$ & 0.983 \\
Alanine aminotransferase (IU/L) & $22(6-49)$ & $20(5-66)$ & 0.684 \\
Lactate dehydrogenase (U/L) & $300(131-797)$ & $281(122-517)$ & 0.715 \\
Creatinine (mg/dL) & $0.81(1.57-0.40)$ & $1.15(0.44-5.50)$ & 0.155 \\
Blood urea nitrogen (mg/dL) & $16.5(6.6-37.2)$ & $15.9(5.2-25.4)$ & 0.769 \\
\hline
\end{tabular}



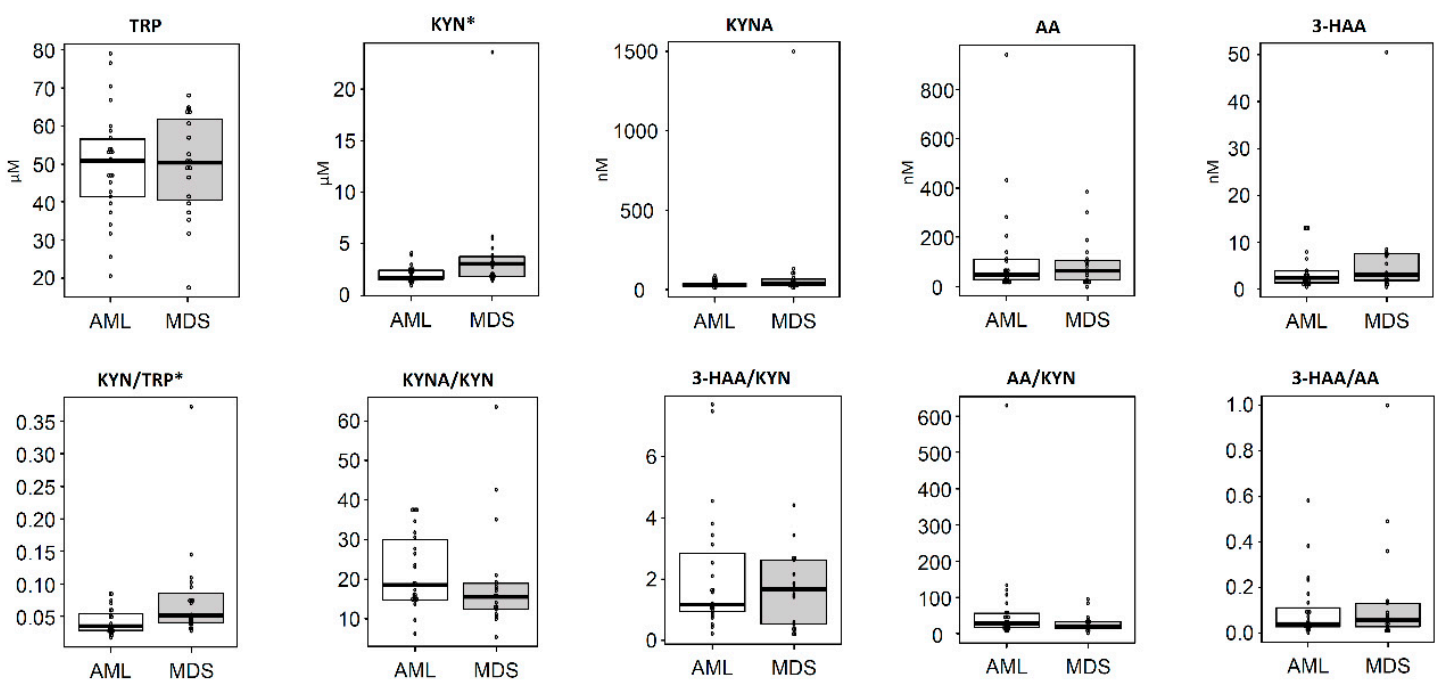

Figure 1. Differences in the concentrations of tryptophan (TRP) metabolites between acute myeloid leukemia (AML) and myelodysplastic syndrome (MDS) patients. Serum TRP and TRP metabolites, such as kynurenine, 3-hydroxyanthranilic acid, kynurenic acid, and anthranilic acid, in samples from AML patients $(n=25)$ and MDS patients $(n=19)$ were analyzed using high-performance liquid chromatography. Statistical analysis was performed using a Welch's $t$-test. The middle line in the box indicates median and the bound indicates $25 \%$ quartile and $75 \%$ quartile. ${ }^{*} p<0.05$. 3-HAA: 3-hydroxyanthranilic acid, AA: anthranilic acid, AML: acute myeloid leukemia, KYN: kynurenine, KYNA: kynurenic acid, MDS: myelodysplastic syndrome, TRP: tryptophan.

\section{Discussion}

We examined the serum TRP levels and the KYN pathway metabolites in patients with hematological malignant tumors. Our study revealed that the serum concentration of KYN was highest in the TRP metabolism pathway among all five diagnostic categories of hematological malignant tumors. In particular, IDO activity was significantly increased in MDS patients. An important limitation of our study is that we did not examine the serum levels of healthy controls. In a recent study, Dong et al. reported an increase in serum KYN in AML patients compared to healthy controls [10]; this is in good agreement with the increase in serum KYN that we observed in all hematological categories. In accordance with previous reports [9], the levels of serum TRP in patients with hematological malignant tumors were lower in females than in males. However, IDO activity showed no differences between men and women. Consequently, the lower TRP levels observed in females may not be due to higher IDO activity.

The KYN pathway is highly regulated in the immune system against cancer, where it promotes TRP metabolism in response to immune activation [3]. IDO expression in the tumor cell induces low levels of TRP and high levels of TRP metabolites in the tumor microenvironment [3]. KYN is generated when IDO is activated in pro-inflammatory microenvironments by inflammatory mediators, such as IFN- $\gamma$ or tumor necrosis factor $\alpha$ [4]. KYN is an endogenous ligand of the aryl hydrocarbon receptor (AHR), which is expressed on naive CD4+ T-cells. Following AHR-KYN engagement, these cells differentiate into Foxp3+ regulatory T-cells [11]. Activation of AHR by KYN on dendritic cells inhibits dendritic cell maturation and immune surveillance, thereby promoting tumor survival [11]. Our results show that KYN and AA concentrations increased, while 3-HAA concentration decreased, in hematological malignant tumors compared to our previous data on healthy volunteers [9]. Moreover, 3-HAA inhibits nitric oxide synthase in macrophages and shows antioxidant activity in response to the local oxidative environment, thus regulating the tissue oxidative status [7]. The biological function of AA remains to be clarified.

Additionally, we compared the concentrations of serum TRP metabolites between MDS and AML patients. Serum KYN was increased in MDS patients. The liver is an important source of blood 
kynurenine metabolites. Chronic renal failure is associated with the accumulation of kynurenine metabolites. Intracellular kynurenine is released when tumor lysis occurs. However, there was no difference in the parameters associated with LDH, liver, and renal function between MDS and AML. IDO activity was also significantly higher in MDS patients than in AML patients, suggesting that the immune system may be more involved in the pathology of MDS than in AML. In this regard, MDS are heterogeneous neoplastic stem cell diseases characterized by bone marrow failure, and recent studies have shown the role of immune dysregulation and innate immune activation in the pathogenesis of MDS [12]. Moreover, the immunomodulatory drug, lenalidomide, has been shown to be effective in some MDS patients [13]. In a recent clinical trial, the serum KYN/TRP ratio was decreased in MDS patients receiving an oral IDO inhibitor [14]. Consequently, the assessment of serum levels of KYN or the KYN/TRP ratio may be useful to predict the therapeutic effect of IDO inhibitors. Taken together, these results suggest that IDO inhibitors may be more suitable for the treatment of MDS than for the treatment of AML.

Cancer immunotherapy has recently produced impressive results in some patients $[15,16]$. An antibody blocking the checkpoint inhibitor PD-1 (programmed cell death protein 1) has been shown to be useful in treating Hodgkin lymphoma patients [15]. Bispecific T-cell engagers and chimeric antigen receptor (CAR) T-cells targeting CD19 have also been approved for B-cell malignant tumors [17]. Approaches to enhancing these responses and predicting who will benefit from them are now needed. The enzymes related to TRP metabolism are potential attractive targets for combinatorial cancer immunotherapy because TRP metabolites affect inflammation, antigen cross-presentation, and overall tolerance [3]. Therefore, some clinical trials are combining IDO inhibitors with chemotherapeutic agents, vaccines, or checkpoint inhibitors to treat solid tumors [18]. A combination of CAR T-cell therapy and IDO inhibition may be effective against these hematological malignant tumors [19]. Moreover, since TRP metabolite levels vary among cases even with the same diagnosis, measurement of TRP metabolites could be a useful method to predict the effectiveness of IDO inhibitors and cancer immune therapy in general. Measuring TRP metabolites in the bone marrow and lymph nodes is the best way to reflect tumor IDO activity; however, in this study, we used serum samples that could be generally obtained. A recent study by Andrea et al. showed that the serum KYN/TRP ratio could represent both a prognostic and a predictive indicator in advanced lung cancer patients treated with checkpoint inhibitors [20]. IDO activity may be involved in the resistance to checkpoint inhibition, and IDO inhibitors may be effective in patients with a high baseline serum KYN/TRP ratio. Patient selection based on these biomarkers can help to avoid negative results in clinical trials designed without considering the immunological background of patients.

Summarily, in this study, we have analyzed the levels of serum TRP and metabolites of the KYN pathway in patients with hematological malignant tumors. KYN presented the highest levels among all diagnostic categories. IDO activity was increased in MDS patients, suggesting that IDO inhibitors might be effective in treating MDS. Further clinical trials are warranted to confirm the role of TRP metabolites as predictive biomarkers as well as therapeutic targets. A better understanding of TRP metabolism will shed light on the abnormal biology of hematological malignant tumors and help to identify the most effective therapeutic approaches to targeting IDO.

Author Contributions: K.Y. and S.N. performed research and contributed to analysis and interpretation of data, statistical analysis, writing the manuscript, and research design; N.N., K.S., M.S., and H.T. contributed to research design and the writing of the manuscript; H.N., T.M., J.K., N.K., T.H., S.F., and Y.Y. performed research and contributed to the analysis and interpretation of data. All authors have read and agreed to the published version of the manuscript.

Funding: This research was funded by MEXT KAKENHI (grant number JP18K15272).

Acknowledgments: The authors would like to thank Chiyoko Sano for her technical assistance.

Conflicts of Interest: The authors declare no conflict of interest. 


\section{References}

1. Uyttenhove, C.; Pilotte, L.; Théate, I.; Stroobant, V.; Colau, D.; Parmentier, N.; Boon, T.; Van Den Eynde, B.J. Evidence for a tumoral immune resistance mechanism based on tryptophan degradation by indoleamine 2,3-dioxygenase. Nat. Med. 2003, 9, 1269-1274. [CrossRef] [PubMed]

2. Cervenka, I.; Agudelo, L.Z.; Ruas, J.L. Kynurenines: Tryptophan's metabolites in exercise, inflammation, and mental health. Science 2017, 357, eaaf9794. [CrossRef] [PubMed]

3. Munn, D.H.; Mellor, A.L. IDO in the Tumor Microenvironment: Inflammation, Counter-Regulation, and Tolerance. Trends Immunol. 2016, 37, 193-207. [CrossRef] [PubMed]

4. $\quad$ Prendergast, G.C.; Mondal, A.; Dey, S.; Laury-Kleintop, L.D.; Muller, A.J. Inflammatory Reprogramming with IDO1 Inhibitors: Turning Immunologically Unresponsive 'Cold' Tumors 'Hot'. Trends Cancer 2018, 4, 38-58. [CrossRef] [PubMed]

5. Ninomiya, S.; Hara, T.; Tsurumi, H.; Hoshi, M.; Kanemura, N.; Goto, N.; Kasahara, S.; Shimizu, M.; Ito, H.; Saito, K.; et al. Indoleamine 2,3-dioxygenase in tumor tissue indicates prognosis in patients with diffuse large B-cell lymphoma treated with R-CHOP. Ann. Hematol. 2011, 90, 409-416. [CrossRef] [PubMed]

6. Ninomiya, S.; Hara, T.; Tsurumi, H.; Goto, N.; Saito, K.; Seishima, M.; Takami, T.; Moriwaki, H. Indoleamine 2,3-dioxygenase expression and serum kynurenine concentrations in patients with diffuse large B-cell lymphoma. Leuk. Lymphoma 2012, 53, 1143-1145. [CrossRef] [PubMed]

7. Stone, T.W.; Stoy, N.; Darlington, L.G. An expanding range of targets for kynurenine metabolites of tryptophan. Trends Pharm. Sci. 2013, 34, 136-143. [CrossRef] [PubMed]

8. Evangelisti, M.; De Rossi, P.; Rabasco, J.; Donfrancesco, R.; Lionetto, L.; Capi, M.; Sani, G.; Simmaco, M.; Nicoletti, F.; Villa, M.P. Changes in serum levels of kynurenine metabolites in paediatric patients affected by ADHD. Eur. Child Adolesc. Psychiatry 2017, 26, 1433-1441. [CrossRef] [PubMed]

9. Sakurai, M.; Yamamoto, Y.; Kanayama, N.; Hasegawa, M.; Mouri, A.; Takemura, M.; Matsunami, H.; Miyauchi, T.; Tokura, T.; Kimura, H.; et al. Serum Metabolic Profiles of the Tryptophan-Kynurenine Pathway in the high risk subjects of major depressive disorder. Sci. Rep. 2020, 10, 1961. [CrossRef] [PubMed]

10. Wang, D.; Tan, G.; Wang, H.; Chen, P.; Hao, J.; Wang, Y. Identification of novel serum biomarker for the detection of acute myeloid leukemia based on liquid chromatography-mass spectrometry. J. Pharm. Biomed. Anal. 2019, 166, 357-363. [CrossRef] [PubMed]

11. Cheong, J.E.; Sun, L. Targeting the IDO1/TDO2-KYN-AhR Pathway for Cancer Immunotherapy-Challenges and Opportunities. Trends Pharmacol. Sci. 2018, 39, 307-325. [CrossRef] [PubMed]

12. Muto, T.; Walker, C.S.; Choi, K.; Hueneman, K.; Smith, M.A.; Gul, Z.; Garcia-Manero, G.; Ma, A.; Zheng, Y.; Starczynowski, D.T. Adaptive response to inflammation contributes to sustained myelopoiesis and confers a competitive advantage in myelodysplastic syndrome HSCs. Nat. Immunol. 2020, 21, 535-545. [CrossRef] [PubMed]

13. Brunner, A.M.; Weng, S.; Cronin, A.; Fathi, A.T.; Habib, A.R.; Stone, R.; Graubert, T.; Steensma, D.P.; Abel, G.A. Impact of lenalidomide use among non-transfusion dependent patients with myelodysplastic syndromes. Am. J. Hematol. 2018, 93, 1119-1126. [CrossRef] [PubMed]

14. Komrokji, R.S.; Wei, S.; Mailloux, A.W.; Zhang, L.; Padron, E.; Sallman, D.; Lancet, J.E.; Tinsley, S.; Nardelli, L.A.; Pinilla-Ibarz, J.; et al. A Phase II Study to Determine the Safety and Efficacy of the Oral Inhibitor of Indoleamine 2,3-Dioxygenase (IDO) Enzyme INCB024360 in Patients with Myelodysplastic Syndromes. Clin. Lymphoma Myeloma Leuk. 2019, 19, 157-161. [CrossRef] [PubMed]

15. Constantinidou, A.; Alifieris, C.; Trafalis, D.T. Targeting Programmed Cell Death -1 (PD-1) and Ligand (PD-L1): A new era in cancer active immunotherapy. Pharmacol. Ther. 2019, 194, 84-106. [CrossRef] [PubMed]

16. Im, A.; Pavletic, S.Z. Immunotherapy in hematologic malignancies: Past, present, and future. J. Hematol. Oncol. 2017, 10, 94. [CrossRef] [PubMed]

17. Chow, V.A.; Shadman, M.; Gopal, A.K. Translating anti-CD19 CAR T-cell therapy into clinical practice for relapsed/refractory diffuse large B-cell lymphoma. Blood 2018, 132, 777-781. [CrossRef] [PubMed]

18. Brochez, L.; Chevolet, I.; Kruse, V. The rationale of indoleamine 2,3-dioxygenase inhibition for cancer therapy. Eur. J. Cancer 2017, 76, 167-182. [CrossRef] [PubMed]

19. Ninomiya, S.; Narala, N.; Huye, L.; Yagyu, S.; Savoldo, B.; Dotti, G.; Heslop, H.E.; Brenner, M.K.; Rooney, C.M.; Ramos, C.A. Tumor indoleamine 2,3-dioxygenase (IDO) inhibits CD19-CAR T cells and is downregulated by lymphodepleting drugs. Blood 2015, 125, 3905-3916. [CrossRef] [PubMed] 
20. Botticelli, A.; Cerbelli, B.; Lionetto, L.; Zizzari, I.; Salati, M.; Pisano, A.; Federica, M.; Simmaco, M.; Nuti, M.; Marchetti, P. Can IDO activity predict primary resistance to anti-PD-1 treatment in NSCLC. J. Transl. Med. 2018, 16, 219. [CrossRef] [PubMed]

Publisher's Note: MDPI stays neutral with regard to jurisdictional claims in published maps and institutional affiliations.

(C) 2020 by the authors. Licensee MDPI, Basel, Switzerland. This article is an open access article distributed under the terms and conditions of the Creative Commons Attribution (CC BY) license (http://creativecommons.org/licenses/by/4.0/). 